\title{
ELEMENTAL AND TOPOGRAPHIC MAPPING OF LAVA FLOW STRUCTURES IN MARE SERENITATIS ON THE MOON
}

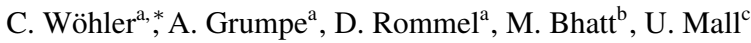 \\ ${ }^{a}$ Image Analysis Group, TU Dortmund University, D-44227 Dortmund, Germany - \\ (christian.woehler, arne.grumpe, daniela.rommel)@tu-dortmund.de \\ ${ }^{\mathrm{b}}$ Space Physics Laboratory, Vikram Sarabhai Space Centre, Thiruvananthapuram, 695022, India - mubhatt19@gmail.com \\ ${ }^{c}$ Max-Planck-Institut für Sonnensystemforschung, Justus-von-Liebig-Weg 3, 37077 Göttingen, Germany - mall@ mps.mpg.de
}

\section{Commission III, ICWG III/II}

KEY WORDS: Topographic mapping, Elemental mapping, Moon, Mare Serenitatis, Lava flow structure

\begin{abstract}
:
The detection of lunar lava flows based on local morphology highly depends on the available images. The thickness of lava flows, however, has been studied by many researchers and lunar lava flows are shown to be as thick as $200 \mathrm{~m}$. Lunar lava flows are supposed to be concentrated on the northwestern lunar nearside. In this study we present elemental abundance maps, a petrological map and a digital terrain model (DTM) of a lava flow structure in northern Mare Serenitatis at $\left(18.0^{\circ} \mathrm{E}, 32.4^{\circ} \mathrm{N}\right)$ and two possible volcanic vents at $\left(11.2^{\circ} \mathrm{E}, 24.6^{\circ} \mathrm{N}\right)$ and $\left(13.5^{\circ} \mathrm{E}, 37.5^{\circ} \mathrm{N}\right)$, respectively. Our abundance maps of the refractory elements Ca, $\mathrm{Mg}$ and our petrological map were obtained based on hyperspectral image data of the Moon Mineralogy Mapper $\left(\mathrm{M}^{3}\right)$ instrument. Our DTM was constructed using GLD100 data in combination with a shape from shading based method to $\mathrm{M}^{3}$ and Lunar Reconnaissance Orbiter (LRO) Narrow Angle Camera (NAC) image data. The obtained NAC-based DEM has a very high effective resolution of about 1-2 $\mathrm{m}$ which comes close to the resolution of the utilized NAC images without requiring intricate processing of NAC stereo image pairs. As revealed by our elemental maps and DEM, the examined lava flow structure occurs on a boundary between basalts consisting of low-Ca/high-Mg pyroxene and high-Ca/low-Mg pyroxene, respectively. The total thickness of the lava flow is about $100 \mathrm{~m}$, which is a relatively large value, but according to our DEM the lava flow may also be composed of two or more layers.
\end{abstract}

\section{INTRODUCTION}

The detection of lunar lava flows based on local morphology highly depends on the available images (Hiesinger et al., 2002). The thickness of lava flows, however, has been studied by many researchers and lunar lava flows are shown to be as thick as $200 \mathrm{~m}$ (e.g. Hiesinger et al., 2002; Gifford and El-Baz, 1981; Neukum and Horn, 1976). Lunar lava flows are supposed to be concentrated on the northwestern lunar nearside (Gifford and El-Baz, 1981). Neither Hiesinger et al. (2002) nor Gifford and El-Baz (1981) found lunar lava flows in Mare Serenitatis.

The detection of lava flows is not at simple task since they are thin structures that do not show a strong shading signature unless the incidence angle of the solar rays is quite large. Hiesinger et al. (2002) presented a method to detect lava flows and estimate the lave flow thickness using crater-size frequency distributions.

In this study we apply a digital terrain model (DTM) refinement method to very high resolution Lunar Reconnaissance Orbiter (LRO) Narrow Angle Camera (NAC) (Chin et al., 2007) images and a the GLD100 DTM (Scholten et al., 2012) of lower resolution to compute high resolution DTMs of a lava flow in Mare Serenitatis. The same technique is applied to two possible volcanic vents. Additionally, elemental abundance maps, and a petrological map of the lava flow structure in northern Mare Serenitatis at $(18.0 \mathrm{E}, 32.7 \mathrm{~N})$ are computed based on hyperspectral image data of the Moon Mineralogy Mapper (M) instrument (Pieters et al., 2009).

\footnotetext{
${ }^{*}$ Corresponding author
}

\section{METHODS}

In order to construct elemental and topographic maps of lava flow structures in the Mare Serenitatis region of the moon, we use several different techniques. This sections briefly reviews the methods applied in this study.

\subsection{Construction of elemental abundance maps}

In order to construct elemental abundance maps, we extract the $\mathrm{M}^{3}$ level-1B radiance data of the region of interest (ROI). The $\mathrm{M}^{3}$ data is resampled to a resolution of 300 pixels per degree which corresponds to about $101 \mathrm{~m}$ per pixel at the lunar equator.

Based on the GLD100 digital terrain model (DTM) (Scholten et al., 2012), a refined DTM of the lunar surface is derived form the $\mathrm{M}^{3}$ data using the methods of Grumpe et al. (2015) and Grumpe and Wöhler (2014). The spectral reflectance of the lunar surface is modeled by the anisotropic multiple scattering approximation Hapke model (Hapke, 2002, 2012) including the unresolved surface roughness model of Hapke (1984). The single-scattering albedo of the Hapke model is considered a free parameter while the remaining parameters are adopted from Warell (2004). Since the GLD100 DTM might miss topographic features smaller than $1.5 \mathrm{~km}$ (Scholten et al., 2012), the single-scattering albedo is derived from the $\mathrm{M}^{3}$ data using the method of Grumpe et al. (2015) with a low-pass filter corresponding to $1.5 \mathrm{~km}$. Based on the computed single-scattering albedo, a refined DTM is constructed using the method of Grumpe and Wöhler (2014). Notably, the refinement of the DTM is derived from the $\mathrm{M}^{3}$ channel 50 which is centered at $1579 \mathrm{~nm}$ which is not influenced by the thermal emis- 
sion spectrum of the surface. The spectral reflectance is inferred from the spectral radiance data by division by the solar irradiance.

The refined DTM is used to infer the local surface temperature using the thermal equilibrium method described in Wöhler et al. (2017). This method iteratively infers the local surface temperature and the local spectral emissivity. Furthermore, we apply the correction for surface roughness of $9^{\circ}$ root-mean-squared slope of Wöhler et al. (2017), i.e. the temperature is modified to an effective temperature of a single black body. The emission spectrum of the black body is the weighted by the inferred spectral emissivity and subtracted from the measured $\mathrm{M}^{3}$ spectral radiance. The thermally corrected spectral radiance is then divided by the solar irradiance yielding the spectral reflectance.

Based on the inferred spectral reflectance and the refined DTM, we compute the spectral single-scattering albedo at full lateral resolution. Inserting the inferred single-scattering albedo and the standard illumination geometry of $30^{\circ}$ incidence angle, $0^{\circ}$ emission angle, and $30^{\circ}$ phase angle (Pieters, 1999) into the Hapke model yields normalized spectral reflectance values.

The spectral parameters of Wöhler et al. (2014) are inferred from the normalized spectral reflectance inserted into the regression model of Bhatt et al. (2015) mapping the spectral parameters onto Lunar Prospector Gamma-Ray Spectrometer (LP GRS) (Lawrence et al., 1998) data. This technique yields elemental abundance maps at the lateral resolution of $\mathrm{M}^{3}$ data.

\subsection{Refinement of digital terrain models}

In this study, we combine the methods of Grumpe et al. (2014) and Grumpe and Wöhler (2014) by simultaneously minimizing

$$
\begin{aligned}
E_{\mathrm{SfS}} & =E_{\mathrm{I}}(p, q)+\gamma E_{\text {int }}\left(p, q, \frac{\partial z}{\partial x}, \frac{\partial z}{\partial y}\right) \\
& +\delta E_{\text {grad }}(p, q)+\tau E_{\text {absDepth }}(z)
\end{aligned}
$$

with respect to $p, q$, and $z$. Following the approach of Horn (1990), we use the variables $p$ and $q$ to denote the photometrically estimated partial derivatives $\frac{\partial z}{\partial x}$ and $\frac{\partial z}{\partial y}$, respectively, of the surface model $z$, i.e. the inclination of the surface as inferred from the image $I$ is not directly related to the surface model $z$. The term $E_{\mathrm{I}}$ describes the integral over the squared intensity deviation between the measured spectral reflectance and the modeled spectral reflectance based on the surface inclination given by $p$ and $q$. This term represents the shading information in the image (Horn, 1990). Since $p$ and $q$ are subject to measurement noise, a surface model $z$ is searched that has a gradient field which is similar to the gradient field given by $p$ and $q$. This integral over the squared norm of the gradient vectors $\left[\frac{\partial z}{\partial x}, \frac{\partial z}{\partial y}\right]$ and $[p, q]$ is the integrability error $E_{\text {int }}$ which relates the surface model $z$ to the shading information (Horn, 1990). To increase the stability and reduce systematic errors, Grumpe et al. (2014) introduced the gradient error $E_{\text {grad }}$ which measures the squared deviation of the photometrically inferred gradient field $p$ and $q$ from the partial derivatives $\frac{\partial z_{\mathrm{DTM}}}{\partial x}$ and $\frac{\partial z_{\mathrm{DTM}}}{\partial y}$ after applying a low-pass filter to each variable, respectively. This error term introduces a soft constraint on the photometrically inferred gradient field. The low-pass filter is introduced to allow small-scale deviations, since the constraining DTM $z_{\mathrm{DTM}}$ is supposed to be of lower lateral resolution. Similarly, Grumpe and Wöhler (2014) introduced $E_{\text {absDepth }}$ which is the integral over the squared deviation between $z$ and $z_{\mathrm{DTM}}$ after applying the low-pass filter to $z$ and $z_{\mathrm{DTM}}$, respectively. The factors $\gamma=10^{-2}, \delta=10^{-2}$, and

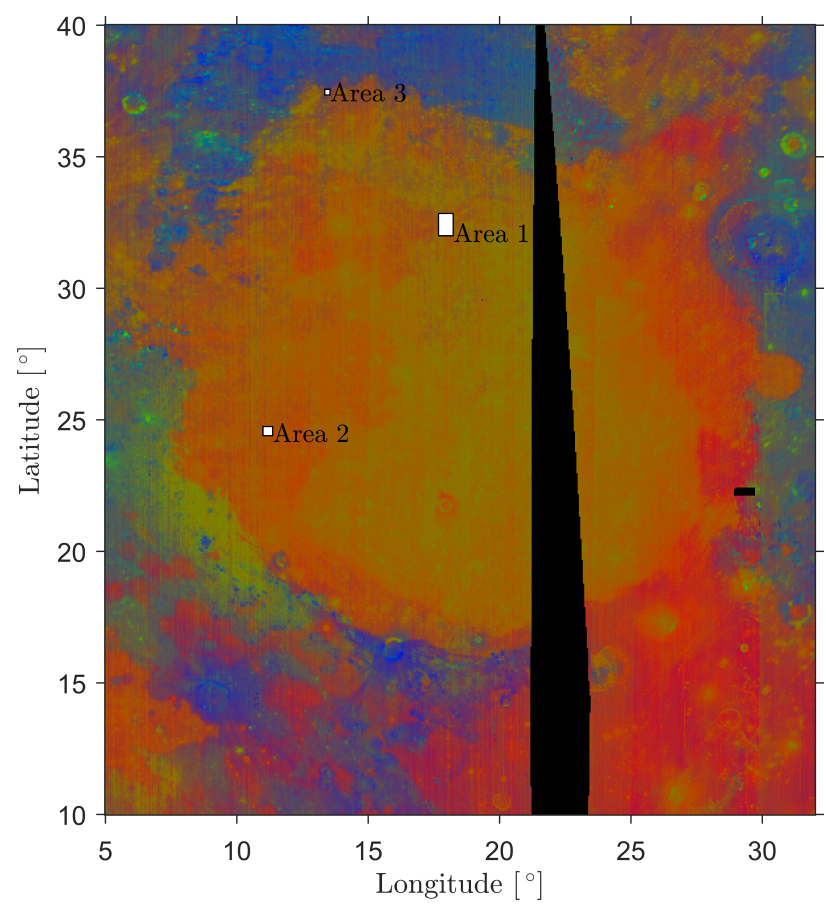

Figure 1: Petrographic map of Mare Serenitatis. The petrographic map is based on the $\mathrm{Fe}$ and $\mathrm{Mg}$ content of the surface following three endmember model of Berezhnoy et al. (2005). The three endmembers are used to create a color composite (Red: basaltic material, Green: Mg-rich rock, Blue: feldspathic material). The three regions considered in this study are shown as white rectangles. Black denotes missing data.

$\tau=10^{2}$ are arbitrary weights. Following the strategy of Horn (1990), we alternate between updating the photometric surface gradient field estimate $p$ and $q$ and the refined DTM $z$.

In order to apply this method to the Lunar Reconnaissance Orbiter Narrow Angle Camera (LROC NAC), we first us the USGS Integrated Software for Imagers and Spectrometers to construct map-projected reflectance images of 1-2 m per pixel resolution. The LROC NAC images are then cropped to the region of interest. Since the GLD100 DTM has a nominal lateral resolution of $100 \mathrm{~m}$ per pixel, we downscale the LROC NAC images by a factor of $2^{6}$ to match the resolution of the GLD100 DTM. Based on this low-resolution LROC NAC image, a refined DTM is computed. The obtained refined DTM is upscaled by a factor of 2 and a corresponding downscaled LROC NAC image is used to compute a further refined DTM. This procedure is repeated until the full LROC NAC resolution is reached.

\section{RESULTS AND DISCUSSION}

Based on an analysis of LP GRS abundance data of the elements $\mathrm{Al}, \mathrm{Fe}$ and $\mathrm{Mg}$, (Berezhnoy et al., 2005) found that the corresponding LP GRS measurements can be described at high accuracy by a plane in the three-dimensional $(\mathrm{Al}, \mathrm{Fe}, \mathrm{Mg})$ abundance space. Consequently, the abundances of $\mathrm{Fe}$ and $\mathrm{Mg}$ alone are sufficient to describe a lunar soil in terms of the petrographic model suggested by (Berezhnoy et al., 2005). This model is defined by the endmembers mare basalt (or pyroxene), Mg-rich rock (e.g. norite), and feldspathic rock. The relative fractions of the three endmembers are read from a ternary diagram in the $\mathrm{Fe}-\mathrm{Mg}$ space and visualized in the form of a composite RGB image. Fig. 1 shows the petrographic map of Mare Serenitatis. We located 
three interesting structures in Mare Serenitatis that were shown in LROC NAC images. The three areas are indicated in Fig. 1. They are captured by the LROC NAC images M181052395LE / RE, M104447576LE / RE, and M1108188835LE / RE, respectively. Area 1 , Area 2 and Area 3 are centered at $\left(18.0^{\circ} \mathrm{E}, 32.4^{\circ}\right.$ $\mathrm{N}),\left(11.2^{\circ} \mathrm{E}, 24.6^{\circ} \mathrm{N}\right)$, and $\left(13.5^{\circ} \mathrm{E}, 37.5^{\circ} \mathrm{N}\right)$, respectively. The LROC NAC images, the GLD100 data and our refined DTMs are shown in Fig. 2. The first area clearly shows a lava flow while the other two areas show possibly volcanic vents. In all cases, the refined DTMs show the same large-scale trends as the GLD100 DTM. However, the spatial resolution is strongly improved. Even small craters are visible within the refined DTMs and all structures occurring in the image may be detected in the refined DTM. Fig. 3-5 show enlarged versions of Fig. 2(c), Fig. 2(f), and Fig. 2(i), respectively. These enlarged versions show that many of the details may not be displayed using the smaller images. The lava flow is found to have a thickness of about $100 \mathrm{~m}$ which is a relatively large value (Hiesinger et al., 2002). The refined DTM suggests that the lava flow consists of at least two layers. The irregularly shaped depression in Area 2 shows a depth of 150-200 m while the possible volcanic vents in Area 3 show an average depth of $100 \mathrm{~m}$.

While the volcanic vents are smaller than $3 \mathrm{~km}$ in extent, the lava flow extends by more than $5 \mathrm{~km}$. Consequently, the volcanic vents are barely visible in the $\mathrm{M}^{3}$ data. The derived elemental abundance maps of $\mathrm{Ca}$ and $\mathrm{Mg}$ of the lava flow in Area 1 are shown in Fig. 6. All other elements did not show differences between the lava flow and the surrounding region. The lava flow clearly shows an increased amount of $\mathrm{Ca}$ while the $\mathrm{Mg}$ content is decreased, corresponding to a composition richer in clinopyroxene than the basalt to the north and south. The strong Ca depletion and $\mathrm{Mg}$ increase occurring at small fresh craters is an artifact of the method resulting from their low optical maturity.

\section{SUMMARY AND CONCLUSION}

In this study, we showed a method to refine DTMs of low lateral resolution, e.g. the GLD100 DTM, using high resolution images, e.g. LROC NAC images. The method produces DTMs of lateral resolution close to the image resolution. All image details can be detected also in the DTM while the low resolution DTM effectively regularizes the trend and thus prevents systematic errors. The possibly volcanic vents do not show exceptional elemental compositions while the lava flow exhibits an increase of the $\mathrm{Ca}$ and a decrease of the $\mathrm{Mg}$ content, corresponding to a composition richer in clinopyroxene than the basalts north and south of the lava flow.

\section{ACKNOWLEDGEMENTS}

This research has made use of the USGS Integrated Software for Imagers and Spectrometers (ISIS).

\section{References}

Berezhnoy, A. A., Hasebe, N., Kobayashi, M., Michael, G. G., Okudaira, O. and Yamashita, N., 2005. A three end-member model for petrologic analysis of lunar prospector gammaray spectrometer data. Planetary and Space Science 53, pp. 1097-1108.

Bhatt, M., Mall, U., Wöhler, C., Grumpe, A. and Bugiolacchi, R., 2015. A comparative study of iron abundance estimation methods: Application to the western nearside of the Moon. Icarus 248, pp. 72-88.

Chin, G., Brylow, S., Foote, M., Garvin, J., Kasper, J., Keller, J., Litvak, M., Mitrofanov, I., Paige, D., Raney, K., Robinson, M., Sanin, A., Smith, D., Spence, H., Spudis, P., Stern, S. A. and Zuber, M., 2007. Lunar Reconnaissance Orbiter Overview: TheInstrument Suite and Mission. Space Science Reviews 129(4), pp. 391-419.

Gifford, A. W. and El-Baz, F., 1981. hicknesses of lunar mare flow fronts. The moon and the planets 24(4), pp. 391-398.

Grumpe, A. and Wöhler, C., 2014. Recovery of elevation from estimated gradient fields constrained by digital elevation maps of lower lateral resolution. ISPRS Journal of Photogrammetry and Remote Sensing 94, pp. 37-54.

Grumpe, A., Belkhir, F. and Wöhler, C., 2014. Construction of lunar DEMs based on reflectance modelling. Advances in Space Research 53(12), pp. 1735-1767.

Grumpe, A., Zirin, V. and Wöhler, C., 2015. A normalisation framework for (hyper-)spectral imagery. Planetary and Space Science 111, pp. 1-33.

Hapke, B., 1984. Bidirectional reflectance spectroscopy: 3. Correction for macroscopic roughness. Icarus 59(1), pp. 41-59.

Hapke, B., 2002. Bidirectional reflectance spectroscopy: 5. The Coherent Backscatter Opposition Effect and Anisotropic Scattering. Icarus $157(2)$, pp. 523-534.

Hapke, B., 2012. Theory of Reflectance and Emittance Spectroscopy. 2nd edn, Cambridge University Press, Cambridge, UK.

Hiesinger, H., Head III, J. W., Wolf, U., Jaumann, R. and Neukum, G., 2002. Lunar mare basalt flow units: Thicknesses determined from crater size-frequency distributions. Geophysical Research Letters 29(8), pp. 89-1-89-4.

Horn, B. K. P., 1990. Height and Gradient from Shading. International Journal of Computer Vision 5(1), pp. 37-75.

Lawrence, D. J., Feldman, W. C., Barraclough, B. L., Binder, A. B., Elphic, R. C., Maurice, S. and Thomsen, D. R., 1998. Global Elemental Maps of the Moon: The Lunar Prospector Gamma-Ray Spectrometer. Science 281, pp. 1484-1489.

Neukum, G. and Horn, P., 1976. Effects of lava flows on lunar crater populations. The moon 15(3), pp. 205-222.

Pieters, C. M., 1999. The Moon as a Spectral Calibration Standard Enabled by Lunar Samples: The Clementine Example. In: Workshop on New Views of the Moon II: Understanding the Moon Through the Integration of Diverse Datasets, Flagstaff, Arizona, p. \#8025.

Pieters, C. M., Boardman, J., Buratti, B., Chatterjee, A., Clark, R., Glavich, T., Green, R., Head III, J., Isaacson, P., Malaret, E., McCord, T., Mustard, J., Petro, N., Runyon, C., Staid, M., Sunshine, J., Taylor, L., Tompkins, S., Varanasi, P. and White, M., 2009. The Moon Mineralogy Mapper $\left(\mathbf{M}^{3}\right)$ on Chandrayaan-1. Current Science 96(4), pp. 500-505.

Scholten, F., Oberst, J., Matz, K.-D., Roatsch, T., Wählisch, M., Speyerer, E. J. and Robinson, M. S., 2012. GLD100: The near-global lunar $100 \mathrm{~m}$ raster DTM from LROC WAC stereo image data. Journal of Geophysical Research.

Warell, J., 2004. Properties of the Hermean regolith: IV. Photometric parameters of Mercury and the Moon contrasted with Hapke modelling. Icarus 167(2), pp. 271-286. 


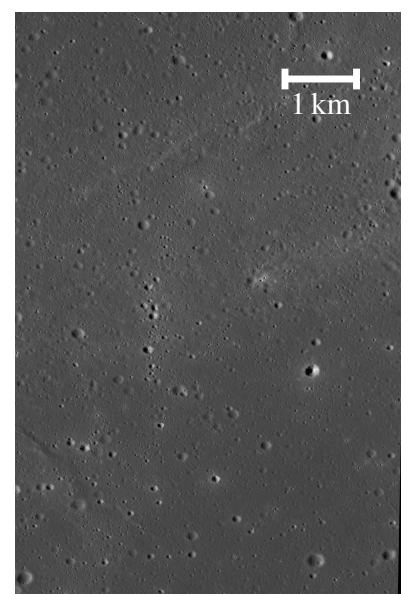

(a) Area 1: LROC NAC

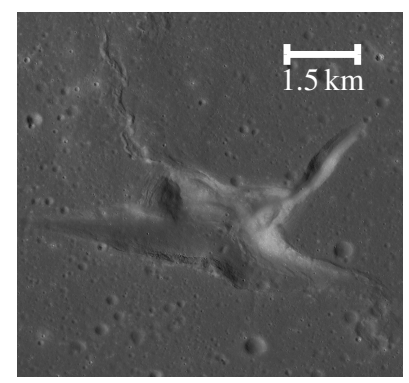

(d) Area 2: LROC NAC

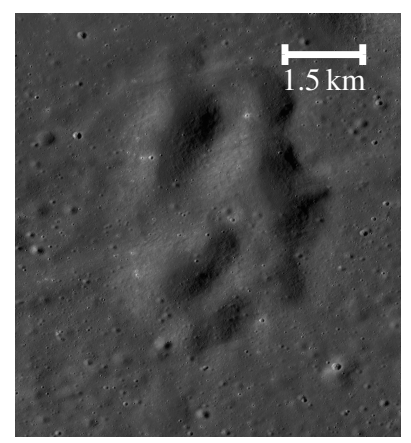

(g) Area 3: LROC NAC



(b) Area 1: GLD100

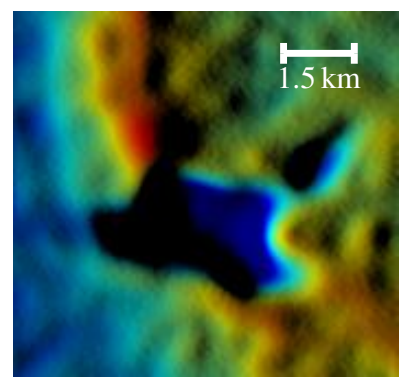

(e) Area 2: GLD100

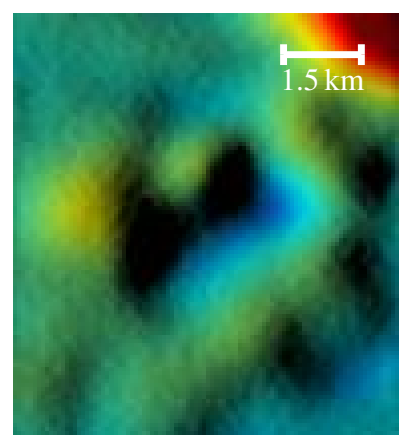

(h) Area 3: GLD100

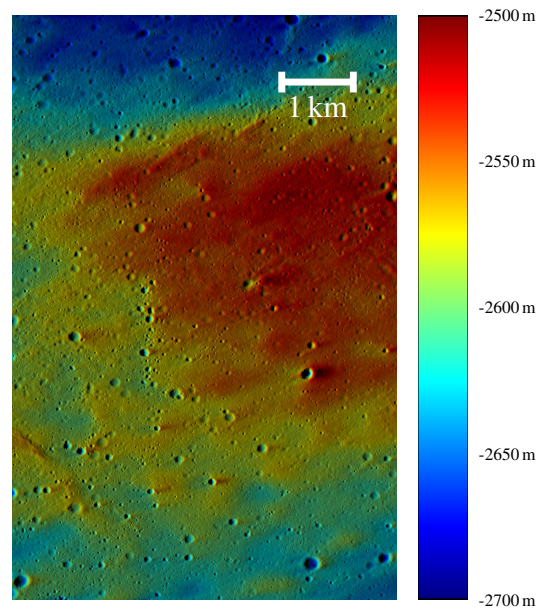

(c) Area 1: Refined DTM

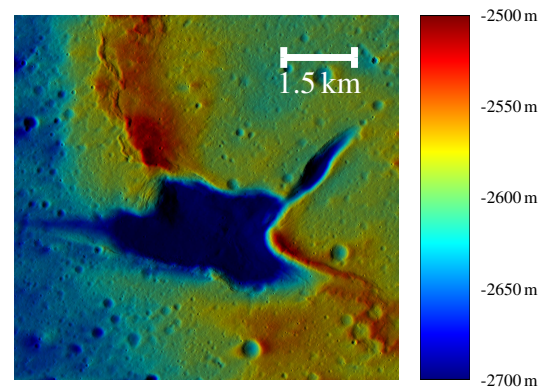

(f) Area 2: Refined DTM

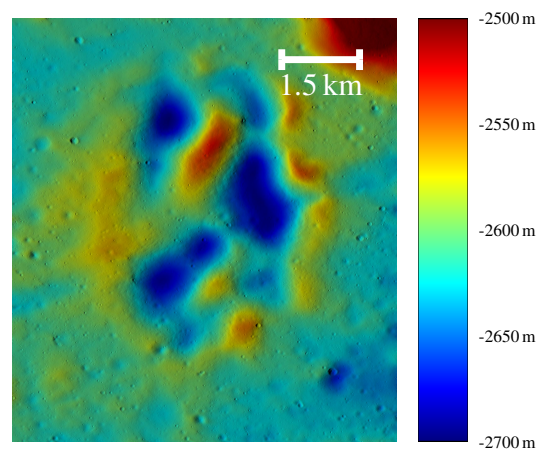

(i) Area 3: Refined DTM

Figure 2: Refined DTMs of the lava flow structures. (a)-(c) LROC NAC image, GLD100 DTM, and the refined DTM of Area 1, respectively. The DTMs clearly show a lava flow of about $100 \mathrm{~m}$ height which is not visible in the image. (d)-(f) LROC NAC image, GLD100 DTM, and the refined DTM of Area 2, respectively. There is a wrinkle ridge in the north-western part of the image and an irregularly shaped depression which is a possible volcanic vent. (g)-(i) LROC NAC image, GLD100 DTM, and the refined DTM of Area 3, respectively. The smooth depression are assumed to be another possibly volcanic vent. In all cases, the refined DTM captures the image details while the GLD100 constrains the solution and successfully reduces systematic errors originating from the integration of a noisy shading information. The visualization of the DTM was computed by color-coding the depth information and weighting the intensity channel of the image by a Lambertian shading of the DTMs, respectively. 




Figure 3: Close-up view of Area 1. 
The International Archives of the Photogrammetry, Remote Sensing and Spatial Information Sciences, Volume XLII-3/W1, 2017 2017 International Symposium on Planetary Remote Sensing and Mapping, 13-16 August 2017, Hong Kong

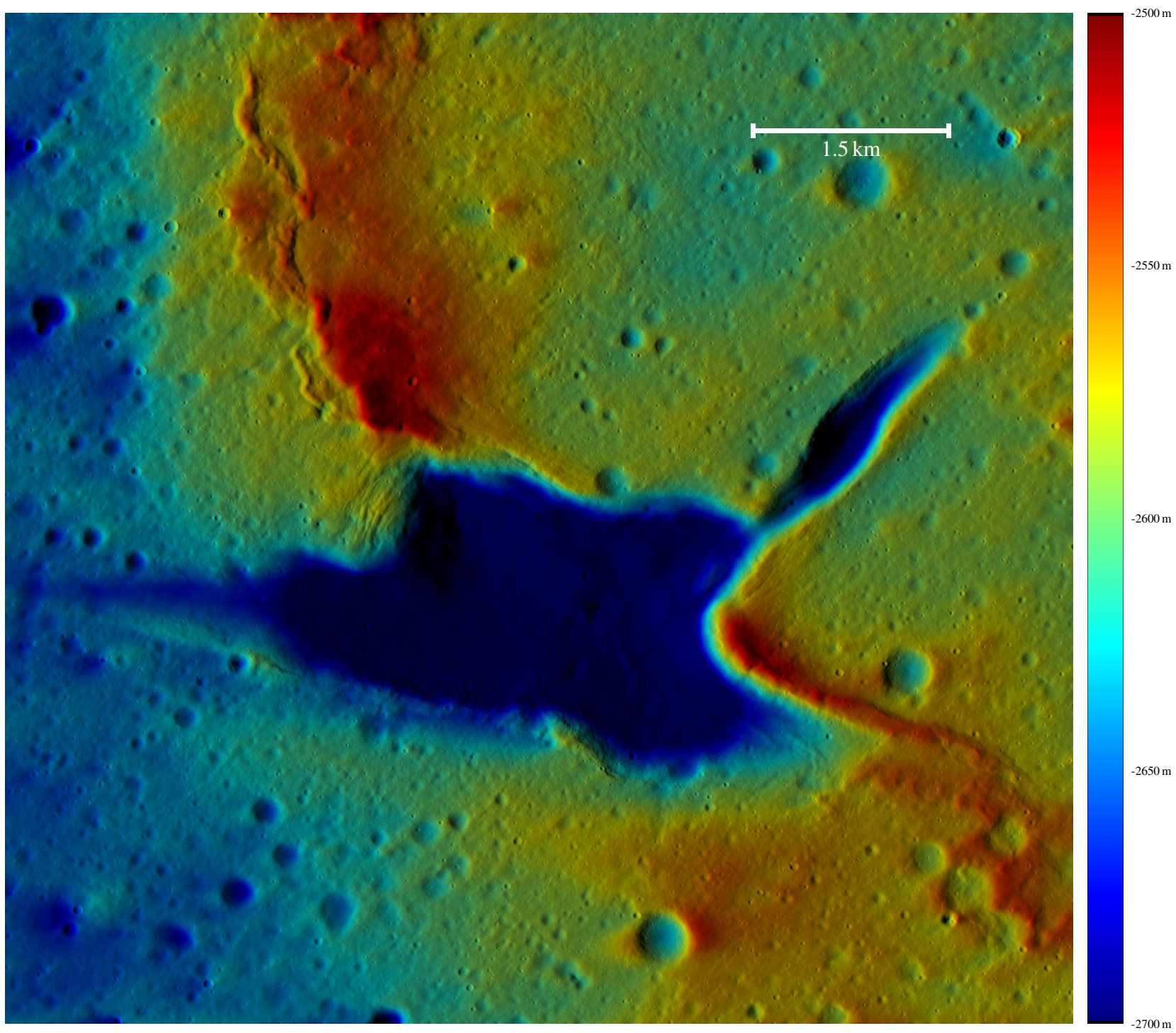

Figure 4: Close-up view of Area 2. 
The International Archives of the Photogrammetry, Remote Sensing and Spatial Information Sciences, Volume XLII-3/W1, 2017 2017 International Symposium on Planetary Remote Sensing and Mapping, 13-16 August 2017, Hong Kong



Figure 5: Close-up view of Area 3. 


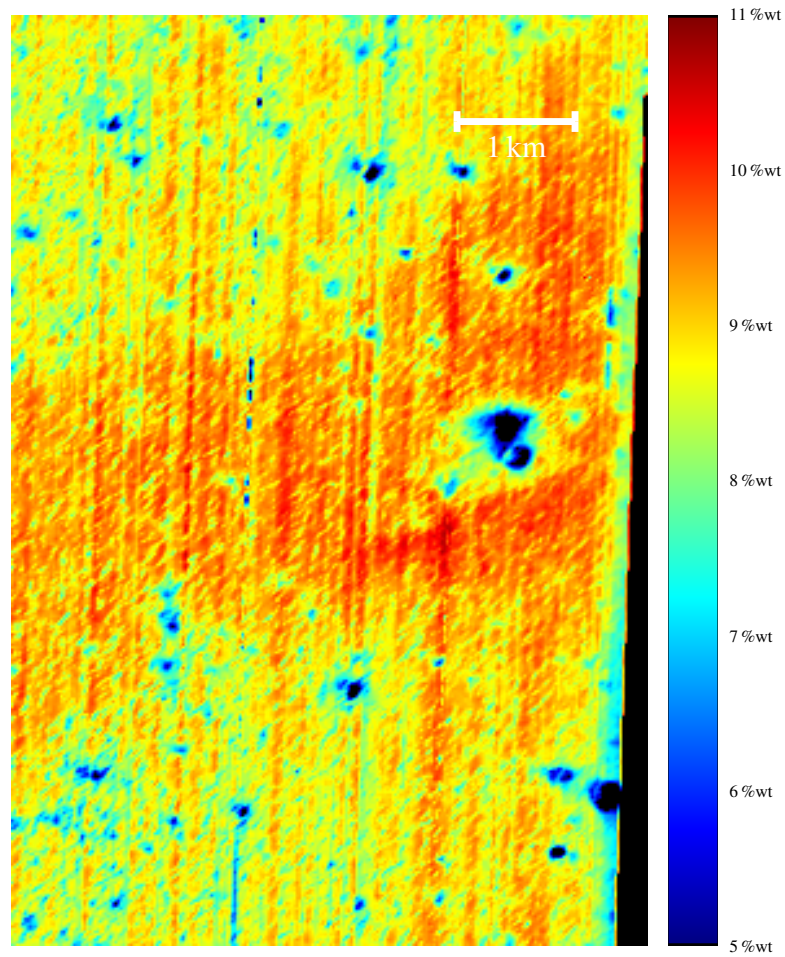

(a) Area 1: $\mathrm{Ca}$

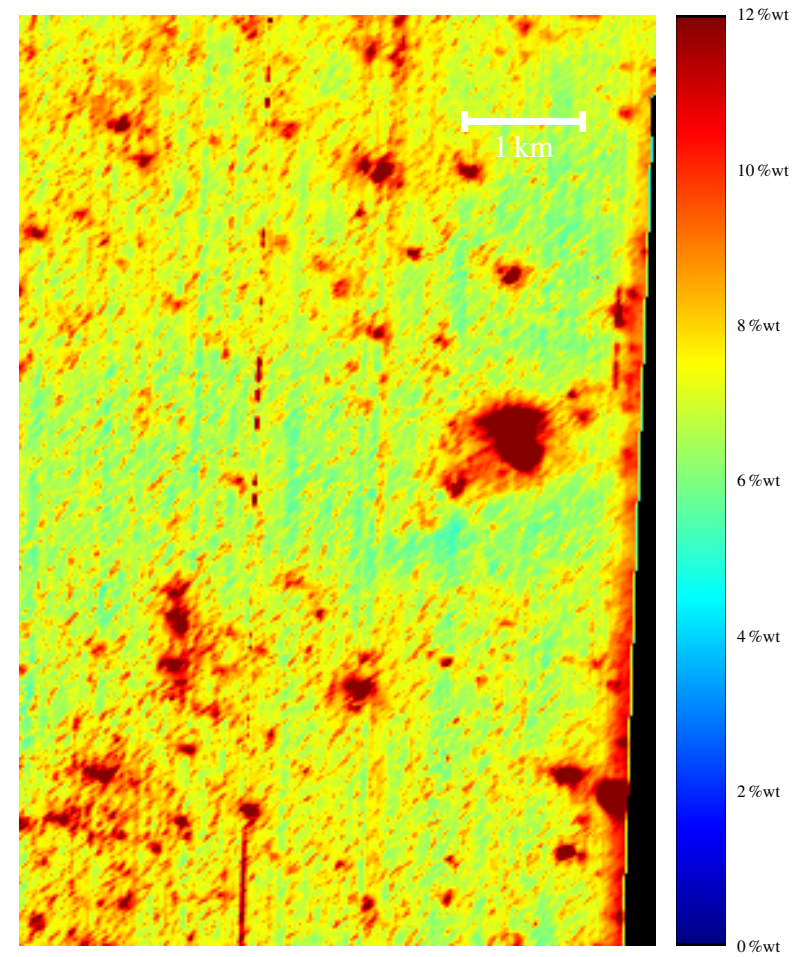

(b) Area 1: $\mathrm{Mg}$

Figure 6: Elemental abundance maps of Area 1. The lava flow shows (a) an enrichment in Ca and (b) a depletion in Mg. Black denotes missing data.

Wöhler, C., Grumpe, A., Berezhnoy, A. A., Feoktistova, E. A., Evdokimova, N. A., Kapoor, K. and Shevchenko, V. V., 2017. Temperature regime and water/hydroxyl behavior in the crater Boguslawsky on the Moon. Icarus 285, pp. 118-136.

Wöhler, C., Grumpe, A., Berezhnoy, A., Bhatt, M. U. and Mall, U., 2014. Integrated topographic and spectral analysis of the lunar surface: Application to impact melt flows and ponds. Icarus 235, pp. 86-122. 